\title{
Optimal Lumbar Lordosis Correction for Adult Spinal Deformity with Severe Sagittal Imbalance in Patients Over Age 60
}

\author{
Role of Pelvic Tilt and Pelvic Tilt Ratio \\ Ki Young Lee, MD, PhD, Jung-Hee Lee, MD, PhD, and Sang-Kyu Im, MD
}

\begin{abstract}
Study Design. A retrospective study.
Objectives. The purpose of this study was to evaluate optimal and ideal target values of the spine balance correction in elderly patients with adult spinal deformity who were over the age of 60 years.
\end{abstract}

Summary of Background Data. The target values of the Scoliosis Research Society -Schwab classification to obtain satisfactory alignment and favorable outcomes are used in many spinal reconstruction surgeries. However, uniformly applying the Scoliosis Research Society-Schwab classification to all elderly patients aged 60 years or older showing sagittal malalignment may lead to several inconsistencies.

Methods. This study included 121 patients (average age $70.5 \mathrm{yr}$ and a minimum 2-yr follow-up) with adult spinal deformity who underwent long-segment fusion from T10 to sacrum. We used Pearson's correlation coefficient to analyze the relationship between clinical and radiographic parameters, and multilinear regression analysis and multivariate logistic regression model (backward elimination method) were conducted using the correlation factors of postoperative (Post) and last follow-up (Last) sagittal vertical axis to find the risk factors of Post sagittal imbalance.

From the Department of Orthopedic Surgery, Graduate School, College of Medicine, Kyung Hee University, Seoul, Korea.

Acknowledgment date: February 24, 2021. Acceptance date: March 26, 2021.

The manuscript submitted does not contain information about medical device(s)/drug(s).

No funds were received in support of this work.

No relevant financial activities outside the submitted work.

This is an open access article distributed under the terms of the Creative Commons Attribution-Non Commercial-No Derivatives License 4.0 (CCBYNC-ND), where it is permissible to download and share the work provided it is properly cited. The work cannot be changed in any way or used commercially without permission from the journal.

Address correspondence and reprint requests to Jung-Hee Lee, MD, PhD, Department of Orthopedic Surgery, Graduate School, College of Medicine, Kyung Hee University, 23, Kyungheedae-ro, Dongdaemun-gu, Seoul 130-

872, Korea; E-mail: ljhspine@gmail.com

DOI: 10.1097/BRS.0000000000004068
Results. Logistic regression analysis with the correlation factors of Post and Last sagittal vertical axis led to risk factors of Post sagittal imbalance, and after confirming the significance of each path, it was confirmed that the effects of pelvic incidence (PI) lumbar lordosis (LL) and Post pelvic tilt ratio (PTr) were valid $(P<0.05)$. After using ROC curve, target value of PI-LL was 1.33, and that of PTr was 25.95\%.

Conclusion. Through our study, the risk factors of Post sagittal imbalance were the Post value of PI-LL and that of PTr, and target value of PI-LL was $<1.33$ and that of PTr was $<25.95 \%$. These target values can be effective guidelines for spine surgeons who perform spine reconstruction surgeries for elderly patients with a pure sagittal imbalance based on Schwab's formula.

Key words: adult spinal deformity, lumbar degenerative kyphosis, lumbar lordosis, pelvic incidence, sagittal balance.

Level of Evidence: 4

Spine 2021;46:E1246-E1253

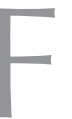

or an optimal surgical correction for adult spinal deformity (ASD) patients, a preoperative (Pre) assessment of postoperative (Post) alignment changes is crucial, and several mathematical prediction formulas and guidelines have been reported for this purpose. ${ }^{1-3}$ The most commonly used alignment target is the Scoliosis Research Society (SRS)-Schwab classification, ${ }^{4}$ owing to the linear regression with Oswestry Disability Index (ODI) measures $(\mathrm{ODI} \geq 40) .^{5}$

However, the assessment of health-related quality of life (HRQOL), including ODI, is relatively subjective. QOL of patients with ASD who undergo long-level constructs with lumbosacral fusion dramatically increases after deformity correction because decompression is appropriately achieved during surgery. ${ }^{6}$ Accordingly, postoperative measures of HRQOL can be overestimated. Furthermore, the ideal lumbar lordosis (LL) that corresponds to SRS-Schwab classification was based on patients with a slightly younger average age $^{7}$; thus, uniformly applying the SRS-Schwab classification to all elderly patients aged 60 years or older showing sagittal malalignment may lead to several inconsistencies. 
Therefore, this study aimed to investigate the optimal and ideal target values of the spine balance correction and an optimal pelvic tilt (PT) based on pelvic incidence (PI) in elderly patients with ASD aged 60 years or older. Focusing on the fact that restoration of normal sagittal alignment is crucial in deformity correction for patients with ASD, we evaluated the patients who were diagnosed with lumbar degenerative kyphosis (LDK), a spinal sagittal malalignment disorder that is relatively common in Asian countries, and who received long-instrumented fusion surgery.

\section{MATERIALS AND METHODS}

\section{Patient Selection}

This study was retrospectively conducted on 121 patients with ASD between 2003 and 2016. The study was approved by the Ethics Committee before data collection.

The inclusion criteria were 1 ) patients aged $\geq 60$ years who had ASD accompanied by sagittal malalignment (sagittal vertical axis $[\mathrm{SVA}]>50 \mathrm{~mm}$, PI minus LL mismatch $>10$, and PT $>25^{\circ}$ ) with a minimum of 2-years follow-up after deformity correction; 2) patients who underwent long segment fixation with setting the uppermost instrumented vertebra at the T10 level and the lowermost instrumented vertebra at the S1 level; 3) patients who clearly showed atrophy of the back musculature on the cross-section area of magnetic resonance imaging and computed tomography scanning as a diagnostic criterion for LDK and clinical signs such as walking difficulty with stooping, inability to lift heavy objects to the front, difficulty in climbing slopes, and the need for elbow support when working in the kitchen, resulting in a hard corn on the extensor surface of the elbow. ${ }^{8}$

\section{Radiographic Measurement}

Sagittal alignment was evaluated using lateral $14 \times 36$-inch full spine radiographs obtained with the patients standing in a neutral unsupported fists-on-clavicle position. ${ }^{9}$ All digital radiographs were evaluated using a validated software (Surgimap, Nemaris Inc, New York, NY). ${ }^{10}$

We evaluated PI, sacral slope, PT, thoracic kyphosis (TK), thoracolumbar junction, LL, lumbosacral junction (LS), and SVA. Sagittal Cobb angles were measured for TK (T5-12), thoracolumbar junction (T10-L2), LL (T12S1), and LS (L4-S1). ${ }^{11,12}$

\section{Clinical Outcome Assessment}

Clinical assessment was performed using the ODI and visual analog scale for back pain and radiating pain. The preoperative, 3-month postoperative, and last follow-up values were compared.

\section{Statistical Analysis}

For continuous variables, analysis of variance with an unpaired $t$ test and Wilcoxon's rank sum test was used. We also used Pearson's correlation coefficient to analyze the relationship between radiographic parameters and clinical outcomes, multilinear regression analysis of these correlation factors led to a predictive formula for Post SVA, and multivariate logistic regression model (backward elimination method) was conducted using variables that were found significant in correlation factors of Post and last follow-up (Last) SVA to find the risk factors of Post sagittal imbalance. All statistical analyses were performed using SPSS software (version 20.0; SPSS Inc, Chicago, IL), and the significance level was set to a $P$ value $<0.05$.

\section{RESULTS}

\section{Baseline Characteristics of the Patients}

The average age at which the surgery was performed was 70.5 years, and the average follow-up period was 79 months. The average body mass index $\left(\mathrm{kg} / \mathrm{m}^{2}\right)$ was 25.1, and BMD $\left(\mathrm{gm} / \mathrm{cm}^{2}\right)$ was 0.932. Sacropelvic fixation with iliac screws was performed in 86 patients. Pedicle subtraction osteotomy was performed in 82 patients, whereas oblique lateral interbody fusion was performed in 27 patients. For L5-S1 fusion, posterior lumbar interbody fusion was carried out in 42 patients and anterior lumbar interbody fusion in 71 patients.

\section{Radiographic Parameters}

The patients showed severe sagittal malalignment before surgery, with a mean PI of $57.9^{\circ}$, SVA of $+189.3 \mathrm{~mm}$, TK of $3.2^{\circ}$, LL of $+1.7^{\circ}$, PI-LL of 60 , and PT of $33.9^{\circ}$ (Table 1 ). After deformity correction, the mean LL correction was $68.1^{\circ}$, and the mean Post PI-LL was -8.6 , with SVA of $-3 \mathrm{~mm}$, TK of $21.4^{\circ}$, LL of $-66.4^{\circ}$, and PT of $15.7^{\circ}$, showing favorable results regarding the spinopelvic parameters. At the final follow-up, sagittal alignment was well maintained, with SVA of $25.9 \mathrm{~mm}$, TK of $33.1^{\circ}$, and PT of $18.5^{\circ}$.

We applied the age-adjusted alignment goals of SVA $\left(2[\right.$ age-55] +25$)$ presented by Lafage et $\mathrm{al}^{13}$ using the mean age of the patients $(70.5 \mathrm{yrs})$; cases in which the SVA was greater than $56 \mathrm{~mm}$ after deformity correction were identified as suboptimal sagittal alignment. The number of patients who showed optimal postoperative sagittal alignment was $110(90.9 \%)$, and the number of patients who revealed suboptimal postoperative sagittal alignment was 11 (9.1\%). Moreover, 99 (81.8\%) patients showed optimal sagittal alignment, and $22(18.2 \%)$ revealed suboptimal sagittal alignment at the final follow-up.

\section{Pelvic Tilt Ratio}

We additionally measured the PT-to-PI ratio (PT ratio, PT/ PI $\times 100 \%$ ) to evaluate the optimal PT based on individual PI values (Table 1). ${ }^{14,15}$ The patients showed Pre PT ratio (PTr) of $58.7 \%$ which decreased after surgery and at the final follow-up to $25.6 \%$ and $29.2 \%$, respectively.

\section{Correlation Between Radiographic Parameters and SVA}

Based on the correlation analysis, Post and Last SVA had negative relationships with TK correction $(r=-0.309$ and -0.317$)$ and SVA correction $(r=-0.585$ and -0.442$)$ 


\begin{tabular}{|c|c|c|c|}
\hline Measurement & Preoperation & Postoperation & Last F/U \\
\hline $\mathrm{PI}\left({ }^{\circ}\right)$ & $57.9 \pm 9.2$ & - & - \\
\hline SS $\left(^{\circ}\right)$ & $24.2 \pm 13.3$ & $44.4 \pm 10.4$ & $43.2 \pm 12.9$ \\
\hline $\mathrm{PT}\left({ }^{\circ}\right)$ & $33.9 \pm 13.2$ & $15.7 \pm 10.5$ & $18.5 \pm 13.6$ \\
\hline PT ratio $(\%)$ & $58.7 \pm 22$ & $25.6 \pm 15.7$ & $29.2 \pm 21.5$ \\
\hline SVA $(\mathrm{mm})$ & $189.3 \pm 69.8$ & $-3 \pm 39.5$ & $25.9 \pm 50.7$ \\
\hline SVA correction & - & $185.6 \pm 92.8$ & - \\
\hline SVA loss & - & - & $28.9 \pm 40.3$ \\
\hline TK $\left(^{\circ}\right)$ & $3.2 \pm 15.7$ & $24.6 \pm 13.3$ & $33.1 \pm 15.5$ \\
\hline $\mathrm{TL}\left({ }^{\circ}\right)$ & $6 \pm 17.8$ & $-19 \pm 16.2$ & $-14.4 \pm 17.5$ \\
\hline $\mathrm{LL}\left({ }^{\circ}\right)$ & $1.7 \pm 18.7$ & $-66.4 \pm 15.5$ & $-62.3 \pm 24.9$ \\
\hline LL correction & - & $68.1 \pm 23.4$ & - \\
\hline LS $\left(^{\circ}\right)$ & $-5.9 \pm 16.5$ & $-25.7 \pm 10.3$ & $-26.2 \pm 13.8$ \\
\hline PI - LL & $59.6 \pm 19.5$ & $-8.6 \pm 14.5$ & $-4.5 \pm 24.7$ \\
\hline ODI & $37.4 \pm 3.3$ & $18.1 \pm 7.6$ & $13.1 \pm 6.5$ \\
\hline VAS for back pain & $7.7 \pm 1.1$ & $3.5 \pm 1.6$ & $2.5 \pm 1.5$ \\
\hline VAS for radiating pain & $8 \pm 0.9$ & $2.3 \pm 1.1$ & $1.2 \pm 1$ \\
\hline
\end{tabular}

and positive relationships with Post values of LS $(r=0.317$ and 0.196), LL ( $r=0.580$ and 0.499), PI-LL $(r=0.739$ and $0.614)$, PT $(r=0.297$ and 0.472$)$, and $\operatorname{PTr}(r=0.295$ and 0.454 ) (Table 2).

\section{Multilinear Regression Analysis for Postoperative SVA}

Multilinear regression analysis of these correlation factors led to a predictive formula for the Post SVA $(r=0.767)$ (Table 3). After establishing the significance of each path, it was noted that the effects of Post PI-LL $(\beta=2.071$, $P<0.001)$, SVA correction $(\beta=-0.170, P<0.001)$, and Post $\operatorname{PTr}(\beta=-1.034, P<0.001)$ were valid on Post SVA.

\section{Multivariate Logistic Regression Analysis for Postoperative Sagittal Malalignment}

We further investigated the risk factors among the correlation factors of Post and Last SVA that cause Post sagittal malalignment (Table 4). Using backward stepwise logistic regression, Post PI-LL (odds ratio, 1.284; 95\% confidence interval, 1.132-1.456; $P<0.001$ ) and Post PTr (odds ratio, 0.878;
95\% confidence interval, $0.798-0.966 ; P=0.008)$ were identified to be crucial risk factors for Post sagittal imbalance.

\section{Target Value of PI-LL and PTr}

The receiver operating characteristic (ROC) curve for Post PI-LL (Figure 1) as a predictor of Post sagittal malalignment yielded an area under the curve of 0.957 (95\% confidence interval, $0.921-0.993 ; P<0.001)$. A cutoff value of 1.33 was associated with $90.9 \%$ sensitivity and $90.9 \%$ specificity for predicting Post sagittal malalignment.

And ROC curve for Post PTr (Figure 2) yielded an area under the curve of 0.720 (95\% confidence interval, $0.555-$ $0.885 ; P=0.016)$. The cutoff value of Post PTr of 25.95 was associated with $72.7 \%$ sensitivity and $59.1 \%$ specificity for predicting Post sagittal malalignment.

\section{DICUSSION}

\section{Deformity Correction Based on PI}

PI is a morphologic parameter that does not change, ${ }^{16}$ and it is the only factor that determines the original spinal shape in

\begin{tabular}{|c|c|c|c|c|c|c|c|}
\hline & SVA cor & TK cor & Po LL & Po PI-LL & Po LS & Po PTr & Po PT \\
\hline Po SVA & $-0.585^{\dagger}$ & $-0.309^{\dagger}$ & $0.580^{\dagger}$ & $0.739^{\dagger}$ & $0.317^{\dagger}$ & $0.295^{\dagger}$ & $0.297^{\dagger}$ \\
\hline Last SVA & $-0.442^{\dagger}$ & $-0.317^{\dagger}$ & $0.499^{\dagger}$ & $0.614^{\dagger}$ & $0.196^{*}$ & $0.454^{\dagger}$ & $0.472^{\dagger}$ \\
\hline SVA cor & & & $-0.430^{\dagger}$ & $-0.429^{\dagger}$ & $-0.280^{\dagger}$ & $-0.345^{\dagger}$ & $-0.323^{\dagger}$ \\
\hline SVA loss & & & - & - & - & $0.304^{\dagger}$ & $0.282^{\dagger}$ \\
\hline
\end{tabular}




\begin{tabular}{|c|c|c|c|c|c|c|}
\hline & \multicolumn{2}{|c|}{ Unstandardized Coefficients } & \multirow[t]{2}{*}{$\begin{array}{c}\text { Standardized } \\
\text { Coefficients } \beta\end{array}$} & \multirow[b]{2}{*}{$\mathbf{T}$} & \multirow[b]{2}{*}{ Significance } & \multirow[b]{2}{*}{ VIF } \\
\hline & B & SE & & & & \\
\hline Constant & 72.888 & 9.170 & & 7.948 & $<0.001$ & \\
\hline Po PI-LL & 2.071 & 0.253 & 0.761 & 8.185 & $<0.001^{\dagger}$ & 2.451 \\
\hline SVA correction & -0.170 & 0.028 & -0.400 & -6.083 & $<0.001^{\dagger}$ & 1.228 \\
\hline Po PTr & -1.034 & 0.225 & -0.411 & -4.600 & $<0.001^{\dagger}$ & 2.269 \\
\hline \multicolumn{7}{|c|}{$\begin{array}{l}{ }^{*} R \text { 0.767; } R^{2} 0.588 ; \text { standard error 25.710; Durbin-Watson } 2.254 . \\
\left.{ }^{\dagger} \text { Statistically significant (P value < } 0.05\right) \text {. } \\
\text { LL indicates lumbar lordosis; Pl, pelvic incidence; Po, postoperative; PTr, pelvic tilt ratio; SE, standard error; SVA, sagittal vertical axis; VIF, variance inflation } \\
\text { factor. }\end{array}$} \\
\hline
\end{tabular}

surgical correction for patients with ASD. ${ }^{17}$ Depending on the value of PI, LL can be hypolordotic, normal lordotic, or hyperlordotic. Therefore, the degree of optimal LL correction should be determined according to the PI of the given individual. Schwab et $a l^{4}$ suggested simplistic formulae $(\mathrm{LL}=\mathrm{PI}+9[ \pm 9])$ to estimate $\mathrm{LL}$ required for a given PI value and attempted to quantify the mismatch between pelvic morphology and the lumbar curvature. However, the ideal LL that corresponded to Schwab's formula was based on patients with a slightly younger average age (mean age, $57 \mathrm{yrs}$ ), and its effect on the risk of mechanical complications is unclear. ${ }^{18} \mathrm{~A}$ multicenter study showed that radiographic and implant-related complications occurred in $31.7 \%$ of patients who underwent surgical correction using Schwab's target values. ${ }^{18,19}$

For that reason, the global alignment and proportion score, a PI-based proportional method of analyzing the sagittal plane predictive of mechanical complications, in patients undergoing surgery for ASD has been reported. ${ }^{18}$ However, the ideal LL and apex of LL changes with PI, ${ }^{20}$ and the concept of lordosis distribution using the lower lordosis (L4-S1)-to-total lordosis (L1-S1) ratio had limitations. Further, other factors such as poor bone quality and underlying diseases have a greater impact on the incidence of mechanical complications in elderly patients; hence, there may be limitations in applying the global alignment and proportion score designed for relatively younger patients aged 18 years or older to elderly patients with ASD. ${ }^{21}$
In addition, we encountered several elderly patients with ASD in which a small LL correction yielded a gradual collapse of the global sagittal balance, resulting in a decreased quality of life. Therefore, we recognized that deformity correction should be performed differently from the previous correction method for patients aged 60 years or older.

\section{The Significance of Sagittal Balance and Lumbar Lordosis Correction}

In this study, unlike the SRS-Schwab classification based on studies correlating HRQOL scores, we focused on Post and Last sagittal parameters because HRQOL scores are subjective measuring methods. Inappropriate correction of sagittal alignment after deformity correction may cause postoperative instability, pain, and mechanical complications. ${ }^{22-25}$ Thus, we applied the mean age of the patients to the SVA formula among the age-adjusted alignment goals presented by Lafage $e t \mathrm{al}^{13}$ and obtained a value of $56 \mathrm{~mm}$. We then focused on determining the factors that caused this Post sagittal imbalance (Post SVA $>56 \mathrm{~mm}$ ). As a result, based on the correlation analysis (Table 2), multilinear regression analysis (Table 3), and logistic regression analysis (Table 4), Post PI-LL and Post PTr were crucial risk factors for Post sagittal malalignment. And according to the ROC curve (Figures 1 and 2), a Post PI-LL cutoff value of 1.33 was associated with $90.9 \%$ sensitivity and $90.9 \%$ specificity, whereas the Post PTr cutoff value of 25.95 was related to

\begin{tabular}{|c|c|c|c|c|c|c|}
\hline & RC & SE & Wald $\chi 2$ & $P$ Value & Odds Ratio & $95 \% \mathrm{Cl}$ \\
\hline Po PI-LL & 0.250 & 0.064 & 15.121 & $<0.001^{*}$ & 1.284 & $1.132-1.456$ \\
\hline Po PTr & -0.130 & 0.049 & 7.143 & $0.008^{*}$ & 0.878 & $0.798-0.966$ \\
\hline Constant & 1.108 & 1.305 & 0.721 & 0.396 & 3.028 & \\
\hline
\end{tabular}


ROC curve

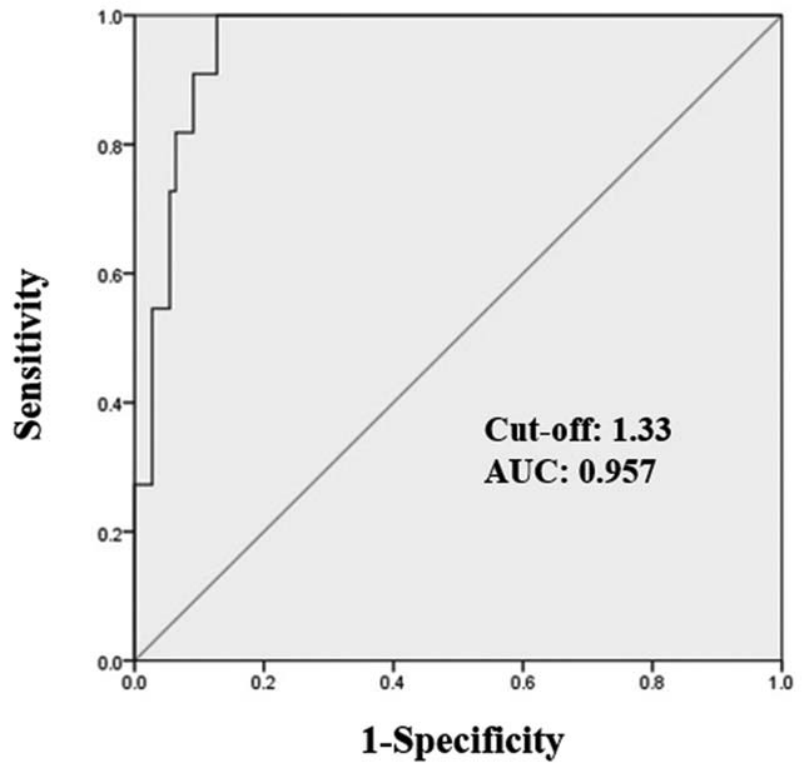

Figure 1. Receiver operating characteristic (ROC) curve analysis was used to determine the cutoff value of PI-LL that predicted postoperative sagittal imbalance. The cutoff value was 1.33. The area under the curve (AUC) was 0.957 , sensitivity was $90.9 \%$, and specificity was $90.9 \%$. PI-LL indicates pelvic incidence-lumbar lordosis.

$72.7 \%$ sensitivity and $59.1 \%$ specificity for predicting sagittal malalignment in postoperative values.

Schwab et $a l^{26,27}$ reported that a progressive loss of LL worsened outcome scores and increased self-reported pain

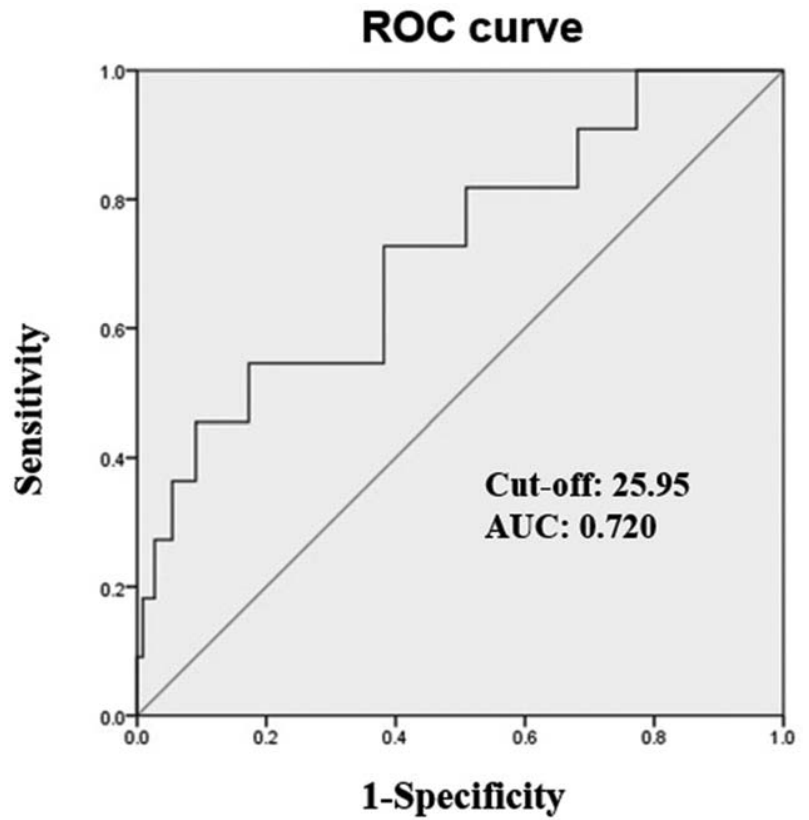

Figure 2. Receiver operating characteristic (ROC) curve analysis to determine the cutoff value of postoperative pelvic tilt ratio (PT/PI $\times$ $100 \%$ ) that predicted postoperative sagittal imbalance. The cutoff value was $25.95 \%$. The area under the curve (AUC) was 0.720, sensitivity was $72.7 \%$, and specificity was $59.1 \%$. PI indicates pelvic incidence; PT, pelvic tilt. and disability. Thus, postoperative LL recovery is an important factor that prompts normal sagittal alignment recovery and prevents decompensation. ${ }^{1,28}$ Therefore, surgeons must decide on the optimal treatment modalities before surgery based on the degrees of LL correction. ${ }^{3,29}$ In this regard, "PILL $\leq 1.33$ " can be an effective guideline for LL correction for patients with ASD over 60 years.

\section{PT Ratio}

In our study, PTr was another significant factor in restoring sagittal balance in patients with ASD over 60 years. Schwab et $\mathrm{al}^{7}$ stated that PT reflected pelvic compensation after a spinal deformity, and PT and quality-of-life measures were shown to have a statistical relationship. However, PT is a posture-dependent measurement, ${ }^{30}$ and patients with a large PI may have considerable PT and sacral slope. Thus, $\mathrm{PT}>20^{\circ}$, while pathologic based on Schwab criteria, may be a natural phenomenon in patients with a large PI. ${ }^{18}$ Therefore, to overcome the limitations of PT in our study, a PTr was calculated along with the measurement of PT.

In a study on 709 asymptomatic adults without spinal pathology, Mac-Thiong et $\mathrm{al}^{15}$ reported that the PTr should be $<50 \%$ in normal adults and that those with a ratio $>50 \%$ can potentially progress with spine pathology. Further, Ferrero et $\mathrm{al}^{14}$ obtained the PTr to evaluate the relationship between spinopelvic parameters and PI, and reported that those with a PTr of $<40 \%$ were considered the low-PT group and those with a PTr of $\geq 40 \%$ were considered the high-PT group. As shown in these studies, several studies have mentioned the significance of PTr; however, the exact reference or target value remains unknown.

In our study, PTr was not correlated with PI before surgery, after surgery, and at the final follow-up; we were able to quantify the pelvic version of all PI values $(r=0.008$, 0.094 , and $0.046 ; P=0.932,0.306$, and 0.613). Hence, we were able to obtain a target value of "PTr $\leq 25.95 \%$ " in our logistic regression and ROC curve for Post sagittal malalignment.

We reclassified our patients according to this target PTr for further study (Table 5); the target value was achieved in 69 of 121 patients. These 69 patients showed smaller SVA and SVA correction loss at last follow-up compared with the remaining 52 patients. Furthermore, LL correction was relatively greater, and Post PI-LL was lower. These results indicate that a larger PTr is associated with the tendency to not maintain sagittal alignment (Figure 3, A-C). PT realignment recovers appropriate femoral pelvic-spinal alignment required for efficient ambulation and is related to walking tolerance ${ }^{31}$; thus, sufficient LL correction results in the lessening of the disability and better maintenance of sagittal alignment, by decreasing Post PTr (Figure 4, A-C). In addition, patients in whom the target PTr was achieved had relatively lower Post and Last ODI compared with those who did not achieve the target PTr, which is consistent with the results of Boissiere et $\mathrm{al}^{18,32}$, which showed that global PT was correlated with the ODI. Therefore, "PTr 


\begin{tabular}{|c|c|c|c|}
\hline Measurement & Post PTr $<26(n=69)$ & Post PTr $>26(n=52)$ & $P$ \\
\hline Pre SVA (mm) & $194.9 \pm 75.9$ & $181.9 \pm 60.6$ & 0.315 \\
\hline Post SVA $(\mathrm{mm})$ & $-9.1 \pm 32.6$ & $5.2 \pm 46.3$ & 0.060 \\
\hline SVA correction $(\mathrm{mm})$ & $204 \pm 83.2$ & $176.7 \pm 81.3$ & 0.074 \\
\hline Last SVA $(\mathrm{mm})$ & $10.7 \pm 33.9$ & $46.1 \pm 61.7$ & $<0.001^{\dagger}$ \\
\hline SVA loss $(\mathrm{mm})$ & $19.8 \pm 35.7$ & $40.8 \pm 43.1$ & $0.004^{\dagger}$ \\
\hline Pre TK $\left(^{\circ}\right)$ & $1.2 \pm 15.3$ & $5.8 \pm 16$ & 0.110 \\
\hline Post TK $\left({ }^{\circ}\right)$ & $25.6 \pm 11.3$ & $23.2 \pm 15.5$ & 0.324 \\
\hline Last TK $\left(^{\circ}\right)$ & $34.3 \pm 13.9$ & $31.7 \pm 17.4$ & 0.363 \\
\hline Pre TL $\left(^{\circ}\right)$ & $6.6 \pm 18.5$ & $5.2 \pm 17$ & 0.653 \\
\hline Post TL $\left(^{\circ}\right)$ & $-19.7 \pm 15.6$ & $-18 \pm 17.1$ & 0.574 \\
\hline Last TL $\left(^{\circ}\right)$ & $-15.5 \pm 16.2$ & $-13.1 \pm 19$ & 0.449 \\
\hline Pre LL $\left(^{\circ}\right)$ & $1.4 \pm 20$ & $2.1 \pm 17.2$ & 0.847 \\
\hline Post LL $\left(^{\circ}\right)$ & $-73.3 \pm 10.8$ & $-57.4 \pm 16.2$ & $<0.001^{\dagger}$ \\
\hline LL correction $\left(^{\circ}\right)$ & $74.7 \pm 21.8$ & $59.4 \pm 22.7$ & $<0.001^{\dagger}$ \\
\hline Post PI-LL & $-15.6 \pm 7.6$ & $0.7 \pm 16.3$ & $<0.001^{\dagger}$ \\
\hline Last LL $\left(^{\circ}\right)$ & $-67 \pm 27.3$ & $-56.1 \pm 20$ & $0.016^{\dagger}$ \\
\hline Pre LS $\left(^{\circ}\right)$ & $-6.4 \pm 17.9$ & $-5.1 \pm 14.5$ & 0.671 \\
\hline Post LS $\left(^{\circ}\right)$ & $-29.3 \pm 9.8$ & $-20.9 \pm 8.9$ & $<0.001^{\dagger}$ \\
\hline Last LS $\left(^{\circ}\right)$ & $-29 \pm 10.3$ & $-22.4 \pm 16.7$ & $0.009^{\dagger}$ \\
\hline $\mathrm{PI}\left({ }^{\circ}\right)$ & $57.7 \pm 9.4$ & $58 \pm 9.1$ & 0.843 \\
\hline Pre SS $\left(^{\circ}\right)$ & $26.5 \pm 14.1$ & $21.1 \pm 11.6$ & $0.024^{\dagger}$ \\
\hline Post SS $\left({ }^{\circ}\right)$ & $50 \pm 7.9$ & $36.9 \pm 8.5$ & $0.000^{\dagger}$ \\
\hline Last SS $\left(^{\circ}\right)$ & $46.5 \pm 13.2$ & $38.8 \pm 11.1$ & $0.001^{\dagger}$ \\
\hline Pre PT $\left(^{\circ}\right)$ & $31.8 \pm 14.1$ & $36.8 \pm 11.6$ & $0.040^{\dagger}$ \\
\hline Post PT $\left({ }^{\circ}\right)$ & $8.8 \pm 5.2$ & $25 \pm 8.4$ & $<0.001^{\dagger}$ \\
\hline Last PT $\left({ }^{\circ}\right)$ & $14.3 \pm 13.4$ & $24.1 \pm 11.9$ & $<0.001^{\dagger}$ \\
\hline Pre PTr (\%) & $54.8 \pm 32.2$ & $63.8 \pm 19.4$ & $0.024^{\dagger}$ \\
\hline Post PTr (\%) & $14.6 \pm 7.9$ & $40.1 \pm 11$ & $<0.001^{\dagger}$ \\
\hline Last $\mathrm{P} \operatorname{Tr}(\%)$ & $22.7 \pm 21.8$ & $37.8 \pm 18.1$ & $<0.001^{\dagger}$ \\
\hline Pre KODI & $37.6 \pm 3.5$ & $37 \pm 3.2$ & 0.350 \\
\hline Post KODI & $16.7 \pm 7.4$ & $20 \pm 7.7$ & $0.018^{\dagger}$ \\
\hline Last KODI & $11.4 \pm 6.3$ & $14.1 \pm 6.4$ & $0.018^{\dagger}$ \\
\hline Pre VAS for back pain & $7.8 \pm 0.9$ & $7.7 \pm 1.3$ & 0.478 \\
\hline Post VAS for back pain & $3 \pm 1.3$ & $4.2 \pm 1.7$ & $<0.001^{\dagger}$ \\
\hline Last VAS for back pain & $2.3 \pm 1.4$ & $2.7 \pm 1.6$ & 0.177 \\
\hline Pre VAS for radiating pain & $8 \pm 0.9$ & $8 \pm 1$ & 0.864 \\
\hline Post VAS for radiating pain & $2.4 \pm 1$ & $2.2 \pm 1.2$ & 0.473 \\
\hline Last VAS for radiating pain & $1.1 \pm 0.8$ & $1.2 \pm 1.1$ & 0.486 \\
\hline \multicolumn{4}{|c|}{$\begin{array}{l}{ }^{*} \text { Data are presented as mean } \pm \text { standard deviation. } \\
{ }^{\dagger} \text { Statistically significant (P value }<0.05 \text { ). } \\
\text { Last indicates last follow-up; LL, lumbar lordosis; LS, lumbosacral junctional angle; ODI, Oswestry disability index; PI, pelvic incidence; Post, postoperative, } \\
\text { Pre, preoperative; PT, pelvic tilt; SS, sacral slope; SVA, sagittal vertical axis; TK, thoracic kyphosis; TL, thoracolumbar junctional angle; VAS, visual analog } \\
\text { scale. }\end{array}$} \\
\hline
\end{tabular}

$\leq 25.95 \%$ " is considered to be another important target value for deformity correction in ASD patients over 60 years.

\section{Limitations}

This study had several limitations. First, owing to its retrospective study design, several variables may exist. Second, our study was conducted on elderly patients aged 60 years or

Spine older; hence, it is difficult to apply our target values to patients aged $<60$ years. Third, while we used the ageadjusted alignment goal presented by Lafage et $a l^{13}$ as a reference for Post sagittal balance, we were unable to take other age-adjusted alignment goals into consideration. In particular, the age-adjusted PI-LL formula showed a value of 10.75 after applying the mean age of our patients; however, our target PI-LL value was 1.33 , which was a 


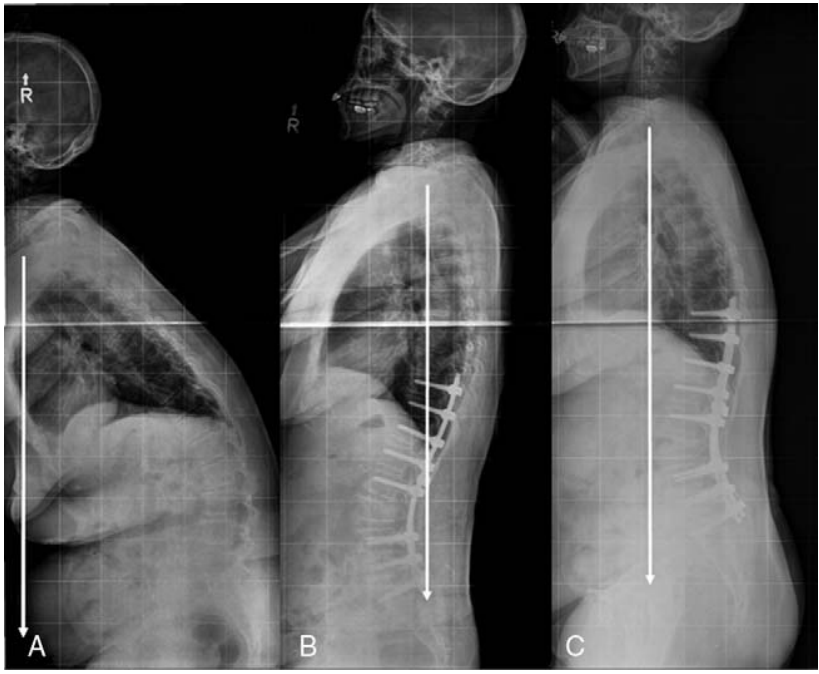

Figure 3. Radiographs showing a 62-year-old female with degenerative lumbar kyphosis who underwent T10-S1 posterior instrumentation with PSO on L3 and PLIF on L4-S1. A, Preoperative whole spine lateral radiograph (SVA, $+252 \mathrm{~mm} ; \mathrm{PI}, 50^{\circ} ; \mathrm{TK}, 9^{\circ} ; \mathrm{LL}, 2^{\circ}$; $\mathrm{PT}$, $24^{\circ}$; PTr, 48; SS, 26 $6^{\circ}$ KODI, 36; VAS of back pain, 6 , and leg pain, 8). B, Postoperative 3-month radiograph showing an optimal sagittal balance with unsatisfactory improvement of PT and PTr (SVA, $-33 \mathrm{~mm}$; TK, $15^{\circ}$; LL, $-43^{\circ}$; PT, 26 ; PTr, 53; SS, 24ㅇ SVA correction, $285 \mathrm{~mm}$; TK correction, $6^{\circ}$; LL correction, 64; KODI, 26; VAS of back pain, 6, and leg pain, 3). C, Postoperative 5-year radiograph showing a suboptimal sagittal balance with unsatisfactory improvement of PT and PTr (SVA, $+81 \mathrm{~mm}$; TK, $32^{\circ}$; $\mathrm{LL},-28^{\circ}$; PT, 30 $0^{\circ}$; $\mathrm{PTr}$, 60; SS, 20 ; SVA loss, $114 \mathrm{~mm}$; LL loss, $15^{\circ}$; KODI, 24; VAS of back pain, 5, and leg pain, 2).

relatively lower value. This difference may be associated with the fact that our patients showed manifestations of a single etiology (LDK). Most patients with LDK are older female adults and show muscle atrophy of the lumbar extensor muscles, subsequent degeneration of the lumbar spine or intervertebral disc, and degenerative change of the lumbosacral facet joint from L2 to S1 level. ${ }^{8,29,33-35}$ Moreover, recently, Yagi et $\mathrm{al}^{36}$ redefined LDK, which showed lumbar kyphosis that occurred abnormally due to degenerative changes in the spine, muscle, and ligament complex, as "drop body syndrome (DBS)." In a study by Yagi et al, ${ }^{36}$ patients with DBS showed a recovery of sagittal balance similar to those without DBS after surgery; however, at 2year follow-up, patients with DBS showed greater loss of global sagittal alignment and higher occurrence of mechanical complications. These results have been attributed to the pathological nature of DBS, and this study is considered to be a crucial result to support the importance of sufficient lordosis correction in LDK patients who were the subjects of our study. In addition, reported studies revealed that sufficient lordosis correction led to clinical and radiological improvements in LDK patients. ${ }^{29}$ Thus, our target values would be useful parameters for deformity correction in patients with pure sagittal imbalance such as patients with LDK, extensor muscle atrophy, and wide-ranging degeneration of the lumbar spine and who are older.

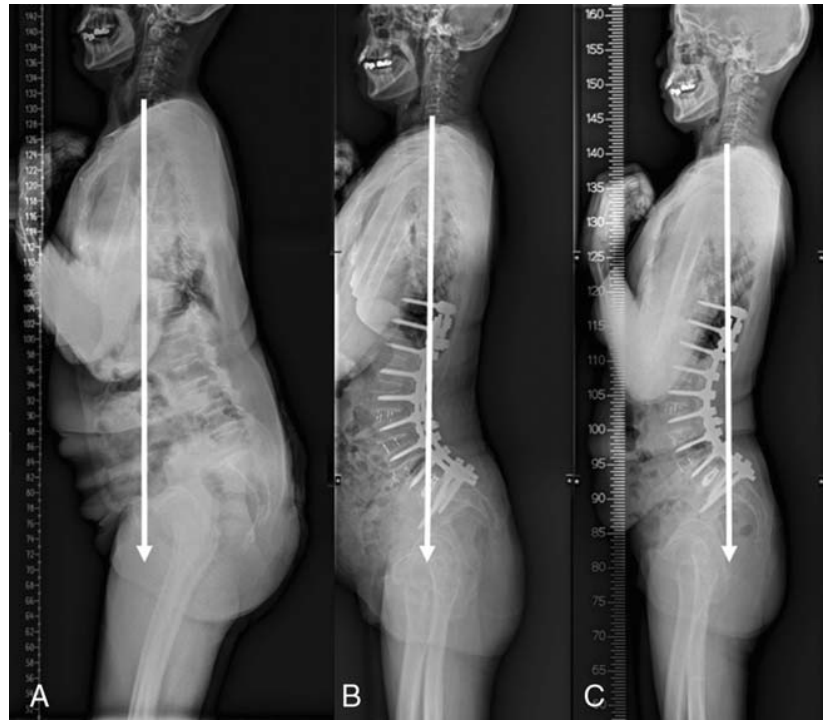

Figure 4. Radiographs showing a 69-year-old female with degenerative lumbar kyphosis who underwent T10-S1 posterior instrumentation with OLIF on L2-5, PLIF on L5-S1, and flexible rod. A, Preoperative whole spine lateral radiograph $\left(\mathrm{SVA},+129 \mathrm{~mm}\right.$; PI, 69 ; TK, $-11^{\circ} ; \mathrm{LL}$, $10^{\circ}$; PT, 46 ; PTr, 67; SS, 23; KODI, 38; VAS of back pain, 6 , and, leg pain, 8). B, Postoperative 3-month radiograph showing an optimal sagittal balance with satisfactory improvement of PT and PTr (SVA, $-2 \mathrm{~mm}$; TK, $7^{\circ}$; $\mathrm{LL},-74^{\circ}$; $\mathrm{PT}, 1^{\circ}$; $\mathrm{PTr}, 17$; SS, 58 ${ }^{\circ}$; SVA correction, $131 \mathrm{~mm}$; TK correction, $18^{\circ}$; LL correction, $84^{\circ}$; KODI, 31; VAS of back pain, 5, and leg pain, 4). C, Postoperative 6-year radiograph showing a well-maintained sagittal balance (SVA, $-6 \mathrm{~mm}$; TK, $16^{\circ}$; $\mathrm{LL},-77^{\circ}$; PT, $19^{\circ}$; PTr, 25; SS, $57^{\circ}$; SVA loss, $-4 \mathrm{~mm}$; LL loss, $-3^{\circ}$; KODI, 18; VAS of back pain, 3 , and, leg pain 2).

\section{CONCLUSION}

The restoration of global sagittal balance in ASD is crucial. In our study, the risk factors of Post sagittal imbalance were the values of Post PI-LL and Post PTr: the target value of Post PI-LL was $<1.33$ and that of Post PTr was $<25.95 \%$. These target values can be effective guidelines for spine surgeons who perform spine reconstruction surgeries for elderly patients with a pure sagittal imbalance based on Schwab's formula.

\section{Key Points}

- In this study, we analyzed the optimal and ideal target values of the spine balance correction and an optimal pelvic tilt based on pelvic incidence in elderly patients with adult spinal deformity aged 6oyears or older.

- The risk factors of Post sagittal imbalance were the Post value of PI-LL and that of PTr, and the target value of PI-LL was $<1.33$ and that of PTr was $<25.95 \%$.

$\square$ Our target values are useful parameters for deformity correction in patients with pure sagittal imbalance such as patients with LDK, extensor muscle atrophy, and wide-ranging degeneration of the lumbar spine and who are older. 


\section{Acknowledgments}

The authors thank Editage (www.editage.co.kr) for English language editing.

\section{References}

1. Kim YJ, Bridwell KH, Lenke LG, et al. An analysis of sagittal spinal alignment following long adult lumbar instrumentation and fusion to L5 or S1: can we predict ideal lumbar lordosis?. Spine (Phila Pa 1976) 2006;31:2343-52.

2. Rose PS, Bridwell KH, Lenke LG, et al. Role of pelvic incidence, thoracic kyphosis, and patient factors on sagittal plane correction following pedicle subtraction osteotomy. Spine (Phila Pa 1976) 2009;34:785-91.

3. Ondra SL, Marzouk S, Koski T, et al. Mathematical calculation of pedicle subtraction osteotomy size to allow precision correction of fixed sagittal deformity. Spine (Phila Pa 1976) 2006;31:E973-9.

4. Schwab F, Ungar B, Blondel B, et al. Scoliosis Research SocietySchwab adult spinal deformity classification: a validation study. Spine (Phila Pa 1976) 2012;37:1077-82.

5. Schwab FJ, Blondel B, Bess S, et al. Radiographical spinopelvic parameters and disability in the setting of adult spinal deformity: a prospective multicenter analysis. Spine (Phila Pa 1976) 2013;38: E803-12.

6. Passias PG, Soroceanu A, Smith J, et al. Postoperative cervical deformity in 215 thoracolumbar patients with adult spinal deformity: prevalence, risk factors, and impact on patient-reported outcome and satisfaction at 2-year follow-up. Spine (Phila Pa 1976) 2015;40:283-91.

7. Schwab F, Lafage V, Patel A, et al. Sagittal plane considerations and the pelvis in the adult patient. Spine (Phila $\mathrm{Pa}$ 1976) 2009;34:1828-33.

8. Takemitsu Y, Harada Y, Iwahara T, et al. Lumbar degenerative kyphosis. Clinical, radiological and epidemiological studies. Spine (Phila Pa 1976) 1988;13:1317-26.

9. Horton WC, Brown CW, Bridwell $\mathrm{KH}$, et al. Is there an optimal patient stance for obtaining a lateral 36" radiograph? A critical comparison of three techniques. Spine (Phila Pa 1976) 2005;30: 427-33.

10. Langella F, Villafane JH, Damilano M, et al. Predictive accuracy of surgimap surgical planning for sagittal imbalance: a cohort study. Spine (Phila Pa 1976) 2017;42:E1297-304.

11. Roussouly P, Pinheiro-Franco JL. Sagittal parameters of the spine: biomechanical approach. Eur Spine J 2011;20 (suppl 5):578-85.

12. Lowe T, Berven SH, Schwab FJ, et al. The SRS classification for adult spinal deformity: building on the King/Moe and Lenke classification systems. Spine (Phila Pa 1976) 2006;31:S119-25.

13. Lafage R, Schwab F, Challier V, et al. Defining spino-pelvic alignment thresholds: should operative goals in adult spinal deformity surgery account for age?. Spine (Phila Pa 1976) 2016;41:62-8.

14. Ferrero E, Vira S, Ames CP, et al. Analysis of an unexplored group of sagittal deformity patients: low pelvic tilt despite positive sagittal malalignment. Eur Spine J 2016;25:3568-76.

15. Mac-Thiong JM, Roussouly P, Berthonnaud E, et al. Age- and sexrelated variations in sagittal sacropelvic morphology and balance in asymptomatic adults. Eur Spine J 2011;20 (suppl 5):572-7.

16. Legaye J, Duval-Beaupere G, Hecquet J, et al. Pelvic incidence: a fundamental pelvic parameter for three-dimensional regulation of spinal sagittal curves. Eur Spine J 1998;7:99-103.

17. Roussouly P, Pinheiro-Franco JL. Biomechanical analysis of the spino-pelvic organization and adaptation in pathology. Eur Spine J 2011;20 (suppl 5):609-18.

18. Yilgor C, Sogunmez N, Boissiere L, et al. Global Alignment and Proportion (GAP) Score: development and validation of a new method of analyzing spinopelvic alignment to predict mechanical complications after adult spinal deformity surgery. J Bone Joint Surg Am 2017;99:1661-72.

19. Soroceanu A, Diebo BG, Burton D, et al. Radiographical and implant-related complications in adult spinal deformity surgery: incidence, patient risk factors, and impact on health-related quality of life. Spine (Phila Pa 1976) 2015;40:1414-21.

20. Lee CS, Chung SS, Park SJ, et al. Simple prediction method of lumbar lordosis for planning of lumbar corrective surgery: radiological analysis in a Korean population. Eur Spine J 2014;23: $192-7$.

21. Noh SH, Ha Y, Obeid I, et al. Modified global alignment and proportion scoring with body mass index and bone mineral density (GAPB) for improving predictions of mechanical complications after adult spinal deformity surgery. Spine J 2020;20:776-84.

22. Glassman SD, Bridwell K, Dimar JR, et al. The impact of positive sagittal balance in adult spinal deformity. Spine (Phila Pa 1976) 2005;30:2024-9.

23. Lazennec JY, Ramare S, Arafati N, et al. Sagittal alignment in lumbosacral fusion: relations between radiological parameters and pain. Eur Spine J 2000;9:47-55.

24. Koike Y, Kotani Y, Terao H, et al. Risk factor analysis of proximal junctional kyphosis after surgical treatment of adult spinal deformity with oblique lateral interbody fusion. Asian Spine J 2021;15: 107-16.

25. Kim SB, Heo YM, Hwang CM, et al. Reliability of the EOS imaging system for assessment of the spinal and pelvic alignment in the sagittal plane. Clin Orthop Surg 2018;10:500-7.

26. Schwab FJ, Smith VA, Biserni M, et al. Adult scoliosis: a quantitative radiographic and clinical analysis. Spine (Phila Pa 1976) 2002;27:387-92.

27. Schwab F, Farcy JP, Bridwell K, et al. A clinical impact classification of scoliosis in the adult. Spine (Phila Pa 1976) 2006;31: 2109-14.

28. Cho KJ, Suk SI, Park SR, et al. Risk factors of sagittal decompensation after long posterior instrumentation and fusion for degenerative lumbar scoliosis. Spine (Phila Pa 1976) 2010;35: 1595-601.

29. Lee JH, Kim KT, Lee SH, et al. Overcorrection of lumbar lordosis for adult spinal deformity with sagittal imbalance: comparison of radiographic outcomes between overcorrection and undercorrection. Eur Spine J 2016;25:2668-75.

30. Neal CJ, McClendon J, Halpin R, et al. Predicting ideal spinopelvic balance in adult spinal deformity. Journal of neurosurgery Spine (Phila Pa 1976) 2011;15:82-91.

31. Schwab F, Patel A, Ungar B, et al. Adult spinal deformity-postoperative standing imbalance: how much can you tolerate? An overview of key parameters in assessing alignment and planning corrective surgery. Spine (Phila Pa 1976) 2010;35:2224-31.

32. Boissiere L, Takemoto M, Bourghli A, et al. Global tilt and lumbar lordosis index: two parameters correlating with health-related quality of life scores-but how do they truly impact disability?. Spine J 2017;17:480-8.

33. Lee JH, Kim JU, Jang JS, et al. Analysis of the incidence and risk factors for the progression of proximal junctional kyphosis following surgical treatment for lumbar degenerative kyphosis: minimum 2-year follow-up. Br J Neurosurg 2014;28:252-8.

34. Lee CS, Lee CK, Kim YT, et al. Dynamic sagittal imbalance of the spine in degenerative flat back: significance of pelvic tilt in surgical treatment. Spine (Phila Pa 1976) 2001;26:2029-35.

35. Lee SH, Kim KT, Suk KS, et al. Sagittal decompensation after corrective osteotomy for lumbar degenerative kyphosis: classification and risk factors. Spine (Phila Pa 1976) 2011;36:E538-44.

36. Yagi M, Fujita N, Okada E, et al. Surgical outcomes for drop body syndrome in adult spinal deformity. Spine (Phila Pa 1976) 2019;44:571-8. 